\title{
Čebyšev's type inequalities for positive linear maps of selfadjoint operators in Hilbert spaces
}

\author{
Silvestru Sever Dragomir
}

\begin{abstract}
Some inequalities for positive linear maps of continuous synchronous (asynchronous) functions of selfadjoint linear operators in Hilbert spaces, under suitable assumptions for the involved operators, are given. Applications for power function and logarithm are provided as well.
\end{abstract}

\section{INTRODUCTION}

We say that the functions $f, g:[a, b] \longrightarrow \mathbb{R}$ are synchronous (asynchronous) on the interval $[a, b]$ if they satisfy the following condition:

$$
(f(t)-f(s))(g(t)-g(s)) \geq(\leq) 0 \text { for each } t, s \in[a, b] .
$$

It is obvious that, if $f, g$ are monotonic and have the same monotonicity on the interval $[a, b]$, then they are synchronous on $[a, b]$ while if they have opposite monotonicity, they are asynchronous.

In 1882-1883, Čebyšev [3] and [4] proved that if the $n$-tuples $\mathbf{a}=\left(a_{1}, \ldots, a_{n}\right)$ and $\mathbf{b}=\left(b_{1}, \ldots, b_{n}\right)$ are monotonic in the same (opposite) sense, then

$$
\frac{1}{P_{n}} \sum_{i=1}^{n} p_{i} a_{i} b_{i}-\frac{1}{P_{n}} \sum_{i=1}^{n} p_{i} a_{i} \frac{1}{P_{n}} \sum_{i=1}^{n} p_{i} b_{i} \geq(\leq) 0,
$$

where $\mathbf{p}=\left(p_{1}, \ldots, p_{n}\right)$ are positive weights.

In the special case $\mathbf{p}=\mathbf{a} \geq \mathbf{0}$, it appears that the inequality (1) has been obtained by Laplace long before Čebyšev (see for example [19, p. 240]).

The inequality (1) was mentioned by Hardy, Littlewood and Pólya in their book [17] in 1934 in the more general setting of synchronous sequences, i.e., if $\mathbf{a}, \mathbf{b}$ are synchronous (asynchronous), this means that

$$
\left(a_{i}-a_{j}\right)\left(b_{i}-b_{j}\right) \geq(\leq) 0 \text { for any } i, j \in\{1, \ldots, n\},
$$

then (1) holds true as well.

2000 Mathematics Subject Classification. Primary: 47A63; Secondary:47A99.

Key words and phrases. Positive linear maps, Selfadjoint operators, Synchronous (asynchronous) functions, Monotonic functions, Čebyšev inequality, Functions of Selfadjoint operators. 
For other recent results on the Čebyšev inequality in either discrete or integral form see $[2,6-10,19,21,22,26-28]$, and the references therein.

The following result provides an inequality of Cebyšev type for functions of selfadjoint operators [14] (see also [13, p. 73] or [15, p. 73]):

Theorem 1.1. Let $A$ be a selfadjoint operator with $\operatorname{Sp}(A) \subseteq[m, M]$ for some real numbers $m<M$. If $f, g:[m, M] \longrightarrow \mathbb{R}$ are continuous and synchronous (asynchronous) on $[m, M]$, then

$$
\langle f(A) g(A) x, x\rangle \geq(\leq)\langle f(A) x, x\rangle\langle g(A) x, x\rangle
$$

for any $x \in H$ with $\|x\|=1$.

Assume that $A$ is a positive operator on the Hilbert space $H$ and $p, q>0$. Then for each $x \in H$ with $\|x\|=1$ we have by (3) the inequality

$$
\left\langle A^{p+q} x, x\right\rangle \geq\left\langle A^{p} x, x\right\rangle\left\langle A^{q} x, x\right\rangle .
$$

If $A$ is positive definite then the inequality (4) also holds for $p, q<0$. If $A$ is positive definite and either $p>0, q<0$ or $p<0, q>0$, then the reverse inequality holds in (4).

Assume that $A$ is positive definite and $p>0$. Then by (3) we have

$$
\left\langle A^{p} \log A x, x\right\rangle \geq\left\langle A^{p} x, x\right\rangle\langle\log A x, x\rangle
$$

for each $x \in H$ with $\|x\|=1$. If $p<0$ then the reverse inequality holds in (5).

The following result that is related to the Čebyšev inequality also holds [14] (see also [13, p. 73] or [15, p. 73]):

Theorem 1.2. Let $A$ be a selfadjoint operator with $\operatorname{Sp}(A) \subseteq[m, M]$ for some real numbers $m<M$.

If $f, g:[m, M] \longrightarrow \mathbb{R}$ are continuous and synchronous on $[m, M]$, then

$$
\begin{aligned}
& \langle f(A) g(A) x, x\rangle-\langle f(A) x, x\rangle\langle g(A) x, x\rangle \\
& \geq[\langle f(A) x, x\rangle-f(\langle A x, x\rangle)][g(\langle A x, x\rangle)-\langle g(A) x, x\rangle]
\end{aligned}
$$

for any $x \in H$ with $\|x\|=1$.

If $f, g$ are asynchronous, then

$$
\begin{aligned}
& \langle f(A) x, x\rangle\langle g(A) x, x\rangle-\langle f(A) g(A) x, x\rangle \\
& \geq[\langle f(A) x, x\rangle-f(\langle A x, x\rangle)][\langle g(A) x, x\rangle-g(\langle A x, x\rangle)]
\end{aligned}
$$

for any $x \in H$ with $\|x\|=1$.

Let $A$ be a selfadjoint operator with $\operatorname{Sp}(A) \subseteq[m, M]$ for some real numbers $m<M$. If $f, g:[m, M] \longrightarrow \mathbb{R}$ are continuous, synchronous and one is convex while the other is concave on $[m, M]$, then by Jensen's inequality for convex (concave) functions and by $(6)$ we have

$$
\begin{aligned}
& \langle f(A) g(A) x, x\rangle-\langle f(A) x, x\rangle\langle g(A) x, x\rangle \\
& \geq[\langle f(A) x, x\rangle-f(\langle A x, x\rangle)][g(\langle A x, x\rangle)-\langle g(A) x, x\rangle] \geq 0
\end{aligned}
$$


for any $x \in H$ with $\|x\|=1$.

If $f, g$ are asynchronous and either both of them are convex or both of them concave on $[m, M]$, then

$$
\begin{aligned}
& \langle f(A) x, x\rangle\langle g(A) x, x\rangle-\langle f(A) g(A) x, x\rangle \\
& \geq[\langle f(A) x, x\rangle-f(\langle A x, x\rangle)][\langle g(A) x, x\rangle-g(\langle A x, x\rangle)] \geq 0
\end{aligned}
$$

for any $x \in H$ with $\|x\|=1$.

Assume that $A$ is a positive operator on the Hilbert space $H$. If $p \in(0,1)$ and $q \in(1, \infty)$, then for each $x \in H$ with $\|x\|=1$ we have the inequality

$$
\begin{aligned}
& \left\langle A^{p+q} x, x\right\rangle-\left\langle A^{p} x, x\right\rangle\left\langle A^{q} x, x\right\rangle \\
& \geq\left[\left\langle A^{q} x, x\right\rangle-\langle A x, x\rangle^{q}\right]\left[\langle A x, x\rangle^{p}-\left\langle A^{p} x, x\right\rangle\right] \geq 0 .
\end{aligned}
$$

If $A$ is positive definite and $p>1, q<0$, then

$$
\begin{aligned}
& \left\langle A^{p} x, x\right\rangle\left\langle A^{q} x, x\right\rangle-\left\langle A^{p+q} x, x\right\rangle \\
& \geq\left[\left\langle A^{q} x, x\right\rangle-\langle A x, x\rangle^{q}\right]\left[\left\langle A^{p} x, x\right\rangle-\langle A x, x\rangle^{p}\right] \geq 0
\end{aligned}
$$

for each $x \in H$ with $\|x\|=1$.

Assume that $A$ is positive definite and $p>1$. Then also

$$
\begin{aligned}
& \left\langle A^{p} \log A x, x\right\rangle-\left\langle A^{p} x, x\right\rangle\langle\log A x, x\rangle \\
& \geq\left[\left\langle A^{p} x, x\right\rangle-\langle A x, x\rangle^{p}\right][\log \langle A x, x\rangle-\langle\log A x, x\rangle] \geq 0
\end{aligned}
$$

for each $x \in H$ with $\|x\|=1$.

Let $H$ be a complex Hilbert space and $\mathcal{B}(H)$, the Banach algebra of bounded linear operators acting on $H$. We denote by $\mathcal{B}^{+}(H)$ the convex cone of all positive operators on $H$ and by $\mathcal{B}^{++}(H)$ the convex cone of all positive definite operators on $H$.

Let $H, K$ be complex Hilbert spaces. Following [5] (see also [30, p. 18]) we can introduce the following definition:

Definition 1.1. A map $\Phi: \mathcal{B}(H) \rightarrow \mathcal{B}(K)$ is linear if it is additive and homogeneous, namely

$$
\Phi(\lambda A+\mu B)=\lambda \Phi(A)+\mu \Phi(B)
$$

for any $\lambda, \mu \in \mathbb{C}$ and $A, B \in \mathcal{B}(H)$. The linear map $\Phi: \mathcal{B}(H) \rightarrow \mathcal{B}(K)$ is positive if it preserves the operator order, i.e. if $A \in \mathcal{B}^{+}(H)$ then $\Phi(A) \in$ $\mathcal{B}^{+}(K)$. We write $\Phi \in \mathfrak{P}[\mathcal{B}(H), \mathcal{B}(K)]$. The linear map $\Phi: \mathcal{B}(H) \rightarrow \mathcal{B}(K)$ is normalised if it preserves the identity operator, i.e. $\Phi\left(1_{H}\right)=1_{K}$. We write $\Phi \in \mathfrak{P}_{N}[\mathcal{B}(H), \mathcal{B}(K)]$.

We observe that a positive linear map $\Phi$ preserves the order relation, namely

$$
A \leq B \text { implies } \Phi(A) \leq \Phi(B)
$$

and preserves the adjoint operation $\Phi\left(A^{*}\right)=\Phi(A)^{*}$. If $\Phi \in \mathfrak{P}_{N}[\mathcal{B}(H), \mathcal{B}(K)]$ and $\alpha 1_{H} \leq A \leq \beta 1_{H}$, then $\alpha 1_{K} \leq \Phi(A) \leq \beta 1_{K}$. 
If the map $\Psi: \mathcal{B}(H) \rightarrow \mathcal{B}(K)$ is linear, positive and $\Psi\left(1_{H}\right) \in \mathcal{B}^{++}(K)$ then by putting $\Phi=\Psi^{-1 / 2}\left(1_{H}\right) \Psi \Psi^{-1 / 2}\left(1_{H}\right)$ we get that $\Phi \in \mathfrak{P}_{N}[\mathcal{B}(H)$, $\mathcal{B}(K)]$, namely it is also normalised.

In the recent paper [25] the following results of Čbyšev type have been obtained:

Theorem 1.3. Let $f, g:[m, M] \longrightarrow \mathbb{R}$ be continuous and synchronous (asynchronous) on $[m, M]$. If $A$ and $B$ are selfadjoint operators with spectra contained in $[m, M]$ and $\Phi \in \mathfrak{P}_{N}[\mathcal{B}(H), \mathcal{B}(K)]$, then for any $x, y \in K$ with $\|x\|=\|y\|=1$ we have

$$
\begin{aligned}
& \langle\Phi(f(A) g(A)) x, x\rangle+\langle\Phi(f(B) g(B)) y, y\rangle \\
& \geq(\leq)\langle\Phi(f(A)) x, x\rangle\langle\Phi(g(B)) y, y\rangle+\langle\Phi(g(A)) x, x\rangle\langle\Phi(f(B)) y, y\rangle
\end{aligned}
$$

In particular, we have the $\check{C}$ ebyšev type inequality

$$
\langle\Phi(f(A) g(A)) x, x\rangle \geq(\leq)\langle\Phi(f(A)) x, x\rangle\langle\Phi(g(A)) x, x\rangle,
$$

for any $x \in K$ with $\|x\|=1$.

Motivated by the above results, we obtain in this paper some new inequalities for positive linear maps of continuous synchronous (asynchronous) functions of selfadjoint linear operators in Hilbert spaces, under suitable assumptions for the involved operators. Applications for power function and logarithm are provided as well.

\section{2. Čebyšev Type Inequalities for Positive Maps}

The following generalization of Theorem 1.3 may be stated:

Theorem 2.1. Let $f, g:[m, M] \longrightarrow \mathbb{R}$ be continuous and synchronous (asynchronous) on $[m, M]$. If $A$ and $B$ are selfadjoint operators with spectra contained in $[m, M]$ and $\Phi, \Psi \in \mathfrak{P}_{N}[\mathcal{B}(H), \mathcal{B}(K)]$, then for any $x, y \in K$ with $\|x\|=\|y\|=1$ we have

$$
\begin{aligned}
& \langle\Phi(f(A) g(A)) x, x\rangle+\langle\Psi(f(B) g(B)) y, y\rangle \\
& \geq(\leq)\langle\Phi(f(A)) x, x\rangle\langle\Psi(g(B)) y, y\rangle+\langle\Phi(g(A)) x, x\rangle\langle\Psi(f(B)) y, y\rangle .
\end{aligned}
$$

In particular, we have (13) and

$$
\begin{aligned}
& \langle\Phi(f(A) g(A)) x, x\rangle+\langle\Psi(f(A) g(A)) y, y\rangle \\
& \geq(\leq)\langle\Phi(f(A)) x, x\rangle\langle\Psi(g(A)) y, y\rangle+\langle\Phi(g(A)) x, x\rangle\langle\Psi(f(A)) y, y\rangle .
\end{aligned}
$$

Proof. We consider only the case of synchronous functions. In this case we have that

$$
f(t) g(t)+f(s) g(s) \geq f(t) g(s)+f(s) g(t)
$$


for each $t, s \in[a, b]$.

Using the continuous functional calculus for the operator $A$ we have

$$
f(A) g(A)+f(s) g(s) 1_{H} \geq g(s) f(A)+f(s) g(A)
$$

for any $s \in[a, b]$.

If we apply to this inequality the positive map $\Phi$ then we get

$$
\Phi(f(A) g(A))+f(s) g(s) 1_{K} \geq g(s) \Phi(f(A))+f(s) \Phi(g(A))
$$

for any $s \in[a, b]$.

Let $x \in K$ with $\|x\|=1$. If we take the inner product in (18), then we get

$$
\begin{aligned}
& \langle\Phi(f(A) g(A)) x, x\rangle+f(s) g(s) \\
& \geq g(s)\langle\Phi(f(A)) x, x\rangle+f(s)\langle\Phi(g(A)) x, x\rangle
\end{aligned}
$$

for any $s \in[a, b]$.

Using the functional calculus for the operator $B$ we get from (19) that

$$
\begin{aligned}
& \langle\Phi(f(A) g(A)) x, x\rangle 1_{H}+f(B) g(B) \\
& \geq\langle\Phi(f(A)) x, x\rangle g(B)+\langle\Phi(g(A)) x, x\rangle f(B)
\end{aligned}
$$

$x \in K$ with $\|x\|=1$.

If we apply to this inequality the positive map $\Psi$ then we get

$$
\begin{aligned}
& \langle\Phi(f(A) g(A)) x, x\rangle 1_{K}+\Psi(f(B) g(B)) \\
& \geq\langle\Phi(f(A)) x, x\rangle \Psi(g(B))+\langle\Phi(g(A)) x, x\rangle \Psi(f(B))
\end{aligned}
$$

for any $x \in K$ with $\|x\|=1$.

Let $y \in K$ with $\|y\|=1$. By taking the inner product in (21) we deduce the desired result (15).

Remark 2.1. If we take in (13) $B=A$, then we get

$$
\begin{aligned}
& \langle\Phi(f(A) g(A)) x, x\rangle+\langle\Phi(f(A) g(A)) y, y\rangle \\
& \geq(\leq)\langle\Phi(f(A)) x, x\rangle\langle\Phi(g(A)) y, y\rangle+\langle\Phi(g(A)) x, x\rangle\langle\Phi(f(A)) y, y\rangle,
\end{aligned}
$$

for any $x, y \in K$ with $\|x\|=\|y\|=1$ and in particular, for $y=x$, in (22) we get the Čebyšev type inequality (14)

$$
\langle\Phi(f(A) g(A)) x, x\rangle \geq(\leq)\langle\Phi(f(A)) x, x\rangle\langle\Phi(g(A)) x, x\rangle .
$$

If the map $\Psi: \mathcal{B}(H) \rightarrow \mathcal{B}(K)$ is linear, positive and $\Psi\left(1_{H}\right) \in \mathcal{B}^{++}(K)$ then $\Phi=\Psi^{-1 / 2}\left(1_{H}\right) \Psi \Psi^{-1 / 2}\left(1_{H}\right)$ is normalised and by (23) we get

$$
\begin{aligned}
& \left\langle\Psi^{-1 / 2}\left(1_{H}\right) \Psi(f(A) g(A)) \Psi^{-1 / 2}\left(1_{H}\right) x, x\right\rangle \\
& \geq(\leq)\left\langle\Psi^{-1 / 2}\left(1_{H}\right) \Psi(f(A)) \Psi^{-1 / 2}\left(1_{H}\right) x, x\right\rangle \\
& \times\left\langle\Psi^{-1 / 2}\left(1_{H}\right) \Psi(g(A)) \Psi^{-1 / 2}\left(1_{H}\right) x, x\right\rangle
\end{aligned}
$$


for any $x \in K$ with $\|x\|=1$.

Moreover, if in (24) we take

$$
x=\frac{1}{\left\|\Psi^{1 / 2}\left(1_{H}\right) v\right\|} \Psi^{1 / 2}\left(1_{H}\right) v, v \in K \text { with } v \neq 0
$$

then we get

(25)

$$
\left\langle\Psi\left(1_{H}\right) v, v\right\rangle\langle\Psi(f(A) g(A)) v, v\rangle \geq(\leq)\langle\Psi(f(A)) v, v\rangle\langle\Psi(g(A)) v, v\rangle
$$

for any $v \in K$.

We also have the following Cauchy-Schwarz' type inequalities:

Corollary 2.1. Let $f:[m, M] \longrightarrow \mathbb{R}$ be continuous on $[m, M]$. If $A$ and $B$ are selfadjoint operators with spectra contained in $[m, M]$ and $\Phi, \Psi \in$ $\mathfrak{P}_{N}[\mathcal{B}(H), \mathcal{B}(K)]$, then for any $x, y \in K$ with $\|x\|=\|y\|=1$ we have

$$
\begin{aligned}
& \left\langle\Phi\left(f^{2}(A)\right) x, x\right\rangle+\left\langle\Psi\left(f^{2}(B)\right) y, y\right\rangle \\
& \geq\langle\Phi(f(A)) x, x\rangle\langle\Psi(f(B)) y, y\rangle+\langle\Phi(f(A)) x, x\rangle\langle\Psi(f(B)) y, y\rangle .
\end{aligned}
$$

In particular, we have for $\Psi=\Phi$, that

$$
\left\langle\Phi\left(f^{2}(A)\right) x, x\right\rangle+\left\langle\Phi\left(f^{2}(B)\right) y, y\right\rangle \geq 2\langle\Phi(f(A)) x, x\rangle\langle\Phi(f(B)) y, y\rangle
$$

and, for $y=x$, we get the following Cauchy-Schwarz inequality

$$
\left\langle\Phi\left(f^{2}(A)\right) x, x\right\rangle \geq\langle\Phi(f(A)) x, x\rangle^{2} .
$$

Assume that $A$ is a positive operator on the Hilbert space $H$ and $p, q>0$. Then for each $x \in H$ with $\|x\|=1$ we have by (23) the inequality

$$
\left\langle\Phi\left(A^{p+q}\right) x, x\right\rangle \geq\left\langle\Phi\left(A^{p}\right) x, x\right\rangle\left\langle\Phi\left(A^{q}\right) x, x\right\rangle .
$$

If $A$ is positive definite then the inequality (29) also holds for $p, q<0$. If $A$ is positive definite and either $p>0, q<0$ or $p<0, q>0$, then the reverse inequality holds in (29).

Assume that $A$ is positive definite and $p>0$. Then by (22) we have

$$
\left\langle\Phi\left(A^{p} \log A\right) x, x\right\rangle \geq\left\langle\Phi\left(A^{p}\right) x, x\right\rangle\langle\Phi(\log A) x, x\rangle
$$

for each $x \in H$ with $\|x\|=1$. If $p<0$ then the reverse inequality holds in (30).

These results generalize the corresponding inequalities from (3)-(5).

Let $P_{j} \in \mathcal{B}(H), j=1, \ldots, k$ be contractions with

$$
\sum_{j=1}^{k} P_{j}^{*} P_{j}=1_{H} .
$$

The map $\Phi: \mathcal{B}(H) \rightarrow \mathcal{B}(H)$ defined by [30]

$$
\Phi(A):=\sum_{j=1}^{k} P_{j}^{*} A P_{j}
$$


is a normalized positive linear map on $\mathcal{B}(H)$.

If $f, g:[m, M] \longrightarrow \mathbb{R}$ are continuous and synchronous (asynchronous) on $[m, M]$ and $A$ is selfadjoint with $\operatorname{Sp}(A) \subseteq[m, M]$, then by (23) we have

$$
\begin{aligned}
& \left\langle\sum_{j=1}^{k} P_{j}^{*} f(A) g(A) P_{j} x, x\right\rangle \\
& \geq(\leq)\left\langle\sum_{j=1}^{k} P_{j}^{*} f(A) P_{j} x, x\right\rangle\left\langle\sum_{j=1}^{k} P_{j}^{*} g(A) P_{j} x, x\right\rangle
\end{aligned}
$$

for each $x \in H$ with $\|x\|=1$.

Assume that $A$ is a positive operator on the Hilbert space $H$ and $p, q>0$. Then for each $x \in H$ with $\|x\|=1$ we have by (32) that

$$
\left\langle\sum_{j=1}^{k} P_{j}^{*} A^{p+q} P_{j} x, x\right\rangle \geq\left\langle\sum_{j=1}^{k} P_{j}^{*} A^{p} P_{j} x, x\right\rangle\left\langle\sum_{j=1}^{k} P_{j}^{*} A^{q} P_{j} x, x\right\rangle
$$

for each $x \in H$ with $\|x\|=1$. If $A$ is positive definite and either $p>0, q<0$ or $p<0, q>0$, then the reverse inequality holds in (33). In this case, by taking the supremum over $x \in H$ with $\|x\|=1$, we get the norm inequality

$$
\left\|\sum_{j=1}^{k} P_{j}^{*} A^{p+q} P_{j}\right\| \leq\left\|\sum_{j=1}^{k} P_{j}^{*} A^{p} P_{j}\right\|\left\|\sum_{j=1}^{k} P_{j}^{*} A^{q} P_{j}\right\|,
$$

where $A$ is positive definite and either $p>0, q<0$ or $p<0, q>0$.

Moreover, by the elementary arithmetic mean-geometric mean inequality, we have

$$
\begin{aligned}
\left\langle\sum_{j=1}^{k} P_{j}^{*} A^{p+q} P_{j} x, x\right\rangle^{1 / 2} & \leq\left\langle\sum_{j=1}^{k} P_{j}^{*} A^{p} P_{j} x, x\right\rangle^{1 / 2}\left\langle\sum_{j=1}^{k} P_{j}^{*} A^{q} P_{j} x, x\right\rangle^{1 / 2} \\
& \leq \frac{1}{2}\left[\left\langle\sum_{j=1}^{k} P_{j}^{*} A^{p} P_{j} x, x\right\rangle+\left\langle\sum_{j=1}^{k} P_{j}^{*} A^{q} P_{j} x, x\right\rangle\right] \\
& =\left\langle\frac{1}{2} \sum_{j=1}^{k} P_{j}^{*}\left(A^{p}+A^{q}\right) P_{j} x, x\right\rangle
\end{aligned}
$$

and by taking the supremum over $x \in H$ with $\|x\|=1$, we get

$$
\left\|\sum_{j=1}^{k} P_{j}^{*} A^{p+q} P_{j}\right\|^{1 / 2} \leq \frac{1}{2}\left\|\sum_{j=1}^{k} P_{j}^{*}\left(A^{p}+A^{q}\right) P_{j}\right\|,
$$

where $A$ is positive definite and either $p>0, q<0$ or $p<0, q>0$. 
Assume that $A$ is positive definite and $p>0$. Then by (32) we have (36)

$$
\left\langle\sum_{j=1}^{k} P_{j}^{*}\left(A^{p} \log A\right) P_{j} x, x\right\rangle \geq\left\langle\sum_{j=1}^{k} P_{j}^{*} A^{p} P_{j} x, x\right\rangle\left\langle\sum_{j=1}^{k} P_{j}^{*}(\log A) P_{j} x, x\right\rangle
$$

for each $x \in H$ with $\|x\|=1$. If $p<0$ then the reverse inequality holds in (36).

If we assume that $A \geq 1_{H}$ and $p<0$, then by (36) we have

$0 \leq\left\langle\sum_{j=1}^{k} P_{j}^{*}\left(A^{p} \log A\right) P_{j} x, x\right\rangle \leq\left\langle\sum_{j=1}^{k} P_{j}^{*} A^{p} P_{j} x, x\right\rangle\left\langle\sum_{j=1}^{k} P_{j}^{*}(\log A) P_{j} x, x\right\rangle$

and by taking the supremum over $x \in H$ with $\|x\|=1$ we get

$$
\left\|\sum_{j=1}^{k} P_{j}^{*}\left(A^{p} \log A\right) P_{j}\right\| \leq\left\|\sum_{j=1}^{k} P_{j}^{*} A^{p} P_{j}\right\|\left\|\sum_{j=1}^{k} P_{j}^{*}(\log A) P_{j}\right\| .
$$

In general, we can state the following norm inequality:

Corollary 2.2. Let $f, g:[m, M] \longrightarrow \mathbb{R}$ be continuous, asynchronous and nonnegative on $[m, M]$. If $A$ and $B$ are selfadjoint operators with spectra contained in $[m, M]$ and $\Phi, \Psi \in \mathfrak{P}_{N}[\mathcal{B}(H), \mathcal{B}(K)]$, then

$$
\begin{aligned}
& \|\Phi(f(A) g(A))\|+\|\Psi(f(B) g(B))\| \\
& \leq\|\Phi(f(A))\|\|\Psi(g(B))\|+\|\Phi(g(A))\|\|\Psi(f(B))\| .
\end{aligned}
$$

In particular, we have

$$
\begin{aligned}
& \|\Phi(f(A) g(A))\|+\|\Phi(f(B) g(B))\| \\
& \leq\|\Phi(f(A))\|\|\Phi(g(B))\|+\|\Phi(g(A))\|\|\Phi(f(B))\|, \\
& \|\Phi(f(A) g(A))\|+\|\Psi(f(A) g(A))\| \\
& \leq\|\Phi(f(A))\|\|\Psi(g(A))\|+\|\Phi(g(A))\|\|\Psi(f(A))\|
\end{aligned}
$$

and

$$
\|\Phi(f(A) g(A))\| \leq\|\Phi(f(A))\|\|\Phi(g(A))\| .
$$

Proof. From the inequality (15) we have

$$
\begin{aligned}
0 & \leq\langle\Phi(f(A) g(A)) x, x\rangle+\langle\Psi(f(B) g(B)) y, y\rangle \\
& \leq\langle\Phi(f(A)) x, x\rangle\langle\Psi(g(B)) y, y\rangle+\langle\Phi(g(A)) x, x\rangle\langle\Psi(f(B)) y, y\rangle,
\end{aligned}
$$

for any $x, y \in K$ with $\|x\|=\|y\|=1$.

Taking the supremum in (42) over $x, y \in K$ with $\|x\|=\|y\|=1$, we get

$$
\begin{aligned}
& \sup _{\|x\|=\|y\|=1}[\langle\Phi(f(A) g(A)) x, x\rangle+\langle\Psi(f(B) g(B)) y, y\rangle] \\
& \leq \sup _{\|x\|=\|y\|=1}[\langle\Phi(f(A)) x, x\rangle\langle\Psi(g(B)) y, y\rangle
\end{aligned}
$$




$$
+\langle\Phi(g(A)) x, x\rangle\langle\Psi(f(B)) y, y\rangle]
$$

Since

$$
\begin{aligned}
& \sup _{\|x\|=\|y\|=1}[\langle\Phi(f(A) g(A)) x, x\rangle+\langle\Psi(f(B) g(B)) y, y\rangle] \\
& =\sup _{\|x\|=1}\langle\Phi(f(A) g(A)) x, x\rangle+\sup _{\|y\|=1}\langle\Psi(f(B) g(B)) y, y\rangle \\
& =\|\Phi(f(A) g(A))\|+\|\Psi(f(B) g(B))\|
\end{aligned}
$$

and

$$
\begin{aligned}
& \sup _{\|x\|=\|y\|=1}[\langle\Phi(f(A)) x, x\rangle\langle\Psi(g(B)) y, y\rangle+\langle\Phi(g(A)) x, x\rangle\langle\Psi(f(B)) y, y\rangle] \\
& \leq \sup _{\|x\|=\|y\|=1}[\langle\Phi(f(A)) x, x\rangle\langle\Psi(g(B)) y, y\rangle] \\
& +\sup _{\|x\|=\|y\|=1}[\langle\Phi(g(A)) x, x\rangle\langle\Psi(f(B)) y, y\rangle] \\
& =\sup _{\|x\|=1}\langle\Phi(f(A)) x, x\rangle \sup _{\|y\|=1}\langle\Psi(g(B)) y, y\rangle \\
& +\sup _{\|x\|=1}\langle\Phi(g(A)) x, x\rangle \sup _{\|y\|=1}\langle\Psi(f(B)) y, y\rangle .
\end{aligned}
$$

We observe that, if $P_{j} \in \mathcal{B}(H), j=1, \ldots, k$ are contractions satisfying condition (31), $f, g:[m, M] \longrightarrow \mathbb{R}$ are continuous, asynchronous and nonnegative on $[m, M]$, then for any $A$ and $B$ selfadjoint operators with spectra contained in $[m, M]$ and $\Phi \in \mathfrak{P}_{N}[\mathcal{B}(H), \mathcal{B}(K)]$ we have the norm inequality (44)

$$
\left\|\sum_{j=1}^{k} P_{j}^{*} \Phi(f(A) g(A)) P_{j}\right\| \leq\left\|\sum_{j=1}^{k} P_{j}^{*} \Phi(f(A)) P_{j}\right\|\left\|\sum_{j=1}^{k} P_{j}^{*} \Phi(g(A)) P_{j}\right\| .
$$

3. Related Results

We have:

Theorem 3.1. Let $f, g:[m, M] \longrightarrow \mathbb{R}$ be continuous and synchronous (asynchronous) on $[m, M]$. If $A$ and $B$ are selfadjoint operators with spectra contained in $[m, M]$ and $\Phi, \Psi \in \mathfrak{P}_{N}[\mathcal{B}(H), \mathcal{B}(K)]$, then for any $x, y \in K$ with $\|x\|=\|y\|=1$ we have

$$
\begin{aligned}
& \langle\Psi(f(B) g(B)) y, y\rangle+f(\langle\Phi(A) x, x\rangle) g(\langle\Phi(A) x, x\rangle) \\
& \geq(\leq) f(\langle\Phi(A) x, x\rangle)\langle\Psi(g(B)) y, y\rangle+g(\langle\Phi(A) x, x\rangle)\langle\Psi(f(B)) y, y\rangle .
\end{aligned}
$$

In particular,

$$
\langle\Phi(f(B) g(B)) y, y\rangle+f(\langle\Phi(A) x, x\rangle) g(\langle\Phi(A) x, x\rangle)
$$




$$
\geq(\leq) f(\langle\Phi(A) x, x\rangle)\langle\Phi(g(B)) y, y\rangle+g(\langle\Phi(A) x, x\rangle)\langle\Phi(f(B)) y, y\rangle
$$

and

$$
\begin{aligned}
& \langle\Psi(f(A) g(A)) y, y\rangle+f(\langle\Phi(A) x, x\rangle) g(\langle\Phi(A) x, x\rangle) \\
& \geq(\leq) f(\langle\Phi(A) x, x\rangle)\langle\Psi(g(A)) y, y\rangle+g(\langle\Phi(A) x, x\rangle)\langle\Psi(f(A)) y, y\rangle .
\end{aligned}
$$

Proof. Since $m 1_{H} \leq A \leq M 1_{H}$, then by taking the map $\Phi$ we get $m 1_{K} \leq$ $\Phi(A) \leq M 1_{K}$. If $x \in K$ with $\|x\|=1$, then $m \leq\langle\Phi(A) x, x\rangle \leq M$.

We consider only the case of synchronous functions. In this case we have that

$$
[f(t)-f(\langle\Phi(A) x, x\rangle)][g(t)-g(\langle\Phi(A) x, x\rangle)] \geq 0
$$

for any $t \in[m, M]$ and $x \in K$ with $\|x\|=1$.

This can be written as

$$
\begin{aligned}
& f(t) g(t)+f(\langle\Phi(A) x, x\rangle) g(\langle\Phi(A) x, x\rangle) \\
& \geq f(\langle\Phi(A) x, x\rangle) g(t)+g(\langle\Phi(A) x, x\rangle) f(t)
\end{aligned}
$$

for any $t \in[m, M]$ and $x \in K$ with $\|x\|=1$.

Fix $x \in K$ with $\|x\|=1$. By utilizing the continuous functional calculus for the operator $B$ we have by (49) that

$$
\begin{aligned}
& f(B) g(B)+f(\langle\Phi(A) x, x\rangle) g(\langle\Phi(A) x, x\rangle) 1_{H} \\
& \geq f(\langle\Phi(A) x, x\rangle) g(B)+g(\langle\Phi(A) x, x\rangle) f(B) .
\end{aligned}
$$

If we take the map $\Psi$ in the inequality (50), we get

$$
\begin{aligned}
& \Psi(f(B) g(B))+f(\langle\Phi(A) x, x\rangle) g(\langle\Phi(A) x, x\rangle) 1_{K} \\
& \geq f(\langle\Phi(A) x, x\rangle) \Psi(g(B))+g(\langle\Phi(A) x, x\rangle) \Psi(f(B)) .
\end{aligned}
$$

If $y \in K$ with $\|y\|=1$, then by taking the inner product in (51) we deduce the desired result (45).

Corollary 3.1. Let $f, g:[m, M] \longrightarrow \mathbb{R}$ be continuous, $A$ a selfadjoint operators with spectrum contained in $[m, M]$ and $\Phi \in \mathfrak{P}_{N}[\mathcal{B}(H), \mathcal{B}(K)]$.

If $f, g$ are synchronous on $[m, M]$, then

$$
\begin{aligned}
& \langle\Phi(f(A) g(A)) x, x\rangle-\langle\Phi(f(A)) x, x\rangle\langle\Phi(g(A)) x, x\rangle \\
& \geq(f(\langle\Phi(A) x, x\rangle)-\langle\Phi(f(A)) x, x\rangle)(\langle\Phi(g(A)) x, x\rangle-g(\langle\Phi(A) x, x\rangle))
\end{aligned}
$$

for any $x \in K$ with $\|x\|=1$.

If $f, g$ are asynchronous on $[m, M]$, then

$$
\begin{aligned}
& \langle\Phi(f(A)) x, x\rangle\langle\Phi(g(A)) x, x\rangle-\langle\Phi(f(A) g(A)) x, x\rangle \\
& \geq(\langle\Phi(f(A)) x, x\rangle-f(\langle\Phi(A) x, x\rangle))(\langle\Phi(g(A)) x, x\rangle-g(\langle\Phi(A) x, x\rangle))
\end{aligned}
$$

for any $x \in K$ with $\|x\|=1$. 
Proof. From the inequality (46) we have for $B=A$ and $y=x$ that

$$
\begin{aligned}
& \langle\Phi(f(A) g(A)) x, x\rangle+f(\langle\Phi(A) x, x\rangle) g(\langle\Phi(A) x, x\rangle) \\
& \geq(\leq) f(\langle\Phi(A) x, x\rangle)\langle\Phi(g(A)) x, x\rangle+g(\langle\Phi(A) x, x\rangle)\langle\Phi(f(A)) x, x\rangle
\end{aligned}
$$

that is equivalent to

$$
\begin{aligned}
& \langle\Phi(f(A) g(A)) x, x\rangle-\langle\Phi(f(A)) x, x\rangle\langle\Phi(g(A)) x, x\rangle \\
& \geq(\leq) f(\langle\Phi(A) x, x\rangle)\langle\Phi(g(A)) x, x\rangle+g(\langle\Phi(A) x, x\rangle)\langle\Phi(f(A)) x, x\rangle \\
& -f(\langle\Phi(A) x, x\rangle) g(\langle\Phi(A) x, x\rangle)-\langle\Phi(f(A)) x, x\rangle\langle\Phi(g(A)) x, x\rangle \\
& =(f(\langle\Phi(A) x, x\rangle)-\langle\Phi(f(A)) x, x\rangle)(\langle\Phi(g(A)) x, x\rangle-g(\langle\Phi(A) x, x\rangle))
\end{aligned}
$$

for any $x \in K$ with $\|x\|=1$.

The case of asynchronous functions goes likewise and the details are omitted.

Remark 3.1. If the map $\digamma: \mathcal{B}(H) \rightarrow \mathcal{B}(K)$ is linear, positive and $\digamma\left(1_{H}\right) \in$ $\mathcal{B}^{++}(K)$ then $\Phi=\digamma^{-1 / 2}\left(1_{H}\right) \digamma \digamma^{-1 / 2}\left(1_{H}\right)$ is normalised and by $(52)$ we have

$$
\begin{aligned}
& \frac{\langle\digamma(f(A)) v, v\rangle}{\left\langle\digamma\left(1_{H}\right) v, v\right\rangle} \frac{\langle\digamma(g(A)) v, v\rangle}{\left\langle\digamma\left(1_{H}\right) v, v\right\rangle}-\frac{\langle\digamma(f(A) g(A)) v, v\rangle}{\left\langle\digamma\left(1_{H}\right) v, v\right\rangle} \\
& \geq\left(f\left(\frac{\langle\digamma(A) v, v\rangle}{\left\langle\digamma\left(1_{H}\right) v, v\right\rangle}\right)-\frac{\langle\digamma(f(A)) v, v\rangle}{\left\langle\digamma\left(1_{H}\right) v, v\right\rangle}\right) \\
& \times\left(\frac{\langle\digamma(g(A)) v, v\rangle}{\left\langle\digamma\left(1_{H}\right) v, v\right\rangle}-g\left(\frac{\langle\digamma(A) v, v\rangle}{\left\langle\digamma\left(1_{H}\right) v, v\right\rangle}\right)\right)
\end{aligned}
$$

for any $v \in K$ with $v \neq 0$, when $f, g$ are synchronous on $[m, M]$, and

$$
\begin{aligned}
& \frac{\langle\digamma(f(A) g(A)) v, v\rangle}{\left\langle\digamma\left(1_{H}\right) v, v\right\rangle}-\frac{\langle\digamma(f(A)) v, v\rangle}{\left\langle\digamma\left(1_{H}\right) v, v\right\rangle} \frac{\langle\digamma(g(A)) v, v\rangle}{\left\langle\digamma\left(1_{H}\right) v, v\right\rangle} \\
& \geq\left(f\left(\frac{\langle\digamma(A) v, v\rangle}{\left\langle\digamma\left(1_{H}\right) v, v\right\rangle}\right)-\frac{\langle\digamma(f(A)) v, v\rangle}{\left\langle\digamma\left(1_{H}\right) v, v\right\rangle}\right) \\
& \times\left(g\left(\frac{\langle\digamma(A) v, v\rangle}{\left\langle\digamma\left(1_{H}\right) v, v\right\rangle}\right)-\frac{\langle\digamma(g(A)) v, v\rangle}{\left\langle\digamma\left(1_{H}\right) v, v\right\rangle}\right)
\end{aligned}
$$

for any $v \in K$ with $v \neq 0$, when $f, g$ are asynchronous on $[m, M]$.

We need the following Jensen's type inequality that has been obtained recently in [16]:

Lemma 3.1. Let $f: I \rightarrow \mathbb{R}$ be a convex function on the interval $I$ and $\Phi$ : $\mathcal{B}(H) \rightarrow \mathcal{B}(K)$ a normalised positive linear map. Then for any selfadjoint operator $A$ whose spectrum $\operatorname{Sp}(A)$ is contained in $I$ we have

$$
f(\langle\Phi(A) y, y\rangle) \leq\langle\Phi(f(A)) y, y\rangle
$$

for any $y \in K,\|y\|=1$. 
Proof. For the sake of completeness, we give here a short proof.

Let $m, M$ with $m<M$ and such that $\operatorname{Sp}(A) \subseteq[m, M] \subset I$. Then $m 1_{H} \leq A \leq M 1_{H}$ and since $\Phi \in \mathfrak{P}_{N}[\mathcal{B}(H), \mathcal{B}(K)]$ we have that $m 1_{K} \leq$ $\Phi(A) \leq M 1_{K}$ showing that $\langle\Phi(A) y, y\rangle \in[m, M]$ for any $y \in K,\|y\|=1$.

By the gradient inequality for the convex function we have for $a=$ $\langle\Phi(A) y, y\rangle \in[m, M]$ that

$$
f(t) \geq f(\langle\Phi(A) y, y\rangle)+(t-\langle\Phi(A) y, y\rangle) f_{+}^{\prime}(\langle\Phi(A) y, y\rangle)
$$

for any $t \in I$, where $f_{+}^{\prime}$ is the right lateral derivative.

Using the continuous functional calculus for the operator $A$ we have for a fixed $y \in K$ with $\|y\|=1$ that

$$
f(A) \geq f(\langle\Phi(A) y, y\rangle) 1_{H}+f_{+}^{\prime}(\langle\Phi(A) y, y\rangle)\left(A-\langle\Phi(A) y, y\rangle 1_{H}\right) .
$$

Since $\Phi \in \mathfrak{P}_{N}[\mathcal{B}(H), \mathcal{B}(K)]$, then by taking the functional $\Phi$ in the inequality $(57)$ we get

$$
\Phi(f(A)) \geq f(\langle\Phi(A) y, y\rangle) 1_{K}+f_{+}^{\prime}(\langle\Phi(A) y, y\rangle)\left(\Phi(A)-\langle\Phi(A) y, y\rangle 1_{K}\right)
$$

for any $y \in K$ with $\|y\|=1$.

This inequality is of interest in itself.

Taking the inner product in (58) we have for any $y \in K$ with $\|y\|=1$ that

$$
\begin{aligned}
& \langle\Phi(f(A)) y, y\rangle \\
& \geq f(\langle\Phi(A) y, y\rangle)\|y\|^{2}+f_{+}^{\prime}(\langle\Phi(A) y, y\rangle)\left(\langle\Phi(A) y, y\rangle-\langle\Phi(A) y, y\rangle\|y\|^{2}\right) \\
& =f(\langle\Phi(A) y, y\rangle)
\end{aligned}
$$

and the inequality (56) is proved.

We can establish now some refinements of the Čebyšev type inequality (23) when some convexity properties are assumed.

Corollary 3.2. Let $f, g:[m, M] \longrightarrow \mathbb{R}$ be continuous, $A$ a selfadjoint operators with spectrum contained in $[m, M]$ and $\Phi \in \mathfrak{P}_{N}[\mathcal{B}(H), \mathcal{B}(K)]$.

If $f, g$ are synchronous on $[m, M]$ and one is convex while the other is concave on $[m, M]$, then

$$
\begin{aligned}
& \langle\Phi(f(A) g(A)) x, x\rangle-\langle\Phi(f(A)) x, x\rangle\langle\Phi(g(A)) x, x\rangle \\
& \geq(f(\langle\Phi(A) x, x\rangle)-\langle\Phi(f(A)) x, x\rangle)(\langle\Phi(g(A)) x, x\rangle-g(\langle\Phi(A) x, x\rangle)) \\
& \geq 0
\end{aligned}
$$

for any $x \in K$ with $\|x\|=1$. 
If $f, g$ are asynchronous and either both of them are convex or both of them concave on $[m, M]$, then

(60)

$$
\begin{aligned}
& \langle\Phi(f(A)) x, x\rangle\langle\Phi(g(A)) x, x\rangle-\langle\Phi(f(A) g(A)) x, x\rangle \\
& \geq(\langle\Phi(f(A)) x, x\rangle-f(\langle\Phi(A) x, x\rangle))(\langle\Phi(g(A)) x, x\rangle-g(\langle\Phi(A) x, x\rangle)) \\
& \geq 0
\end{aligned}
$$

for any $x \in K$ with $\|x\|=1$.

Let $\Phi \in \mathfrak{P}_{N}[\mathcal{B}(H), \mathcal{B}(K)]$ and assume that $A$ is a positive operator on the Hilbert space $H$. If $p \in(0,1)$ and $q \in(1, \infty)$, then for each $x \in K$ with $\|x\|=1$ we have the inequality

$$
\begin{aligned}
& \left\langle\Phi\left(A^{p+q}\right) x, x\right\rangle-\left\langle\Phi\left(A^{p}\right) x, x\right\rangle\left\langle\Phi\left(A^{q}\right) x, x\right\rangle \\
& \geq\left[\left\langle\Phi\left(A^{q}\right) x, x\right\rangle-\langle\Phi(A) x, x\rangle^{q}\right]\left[\langle\Phi(A) x, x\rangle^{p}-\left\langle\Phi\left(A^{p}\right) x, x\right\rangle\right] \geq 0 .
\end{aligned}
$$

If $A$ is positive definite and $p>1, q<0$ then

$$
\begin{aligned}
& \left\langle\Phi\left(A^{p}\right) x, x\right\rangle\left\langle\Phi\left(A^{q}\right) x, x\right\rangle-\left\langle\Phi\left(A^{p+q}\right) x, x\right\rangle \\
& \geq\left[\left\langle\Phi\left(A^{q}\right) x, x\right\rangle-\langle\Phi(A) x, x\rangle^{q}\right]\left[\left\langle\Phi\left(A^{p}\right) x, x\right\rangle-\langle\Phi(A) x, x\rangle^{p}\right] \geq 0
\end{aligned}
$$

for all $x \in K$ with $\|x\|=1$.

Assume that $A$ is positive definite and $p>1$. Then

$$
\begin{aligned}
& \left\langle\Phi\left(A^{p} \log A\right) x, x\right\rangle-\left\langle\Phi\left(A^{p}\right) x, x\right\rangle\langle\Phi(\log A) x, x\rangle \\
& \geq\left[\left\langle\Phi\left(A^{p}\right) x, x\right\rangle-\langle\Phi(A) x, x\rangle^{p}\right][\log \langle\Phi(A) x, x\rangle-\langle\Phi(\log A) x, x\rangle] \geq 0
\end{aligned}
$$

for all $x \in K$ with $\|x\|=1$.

These results generalize the corresponding inequalities from (10)-(12).

\section{REFERENCES}

[1] M. Biernacki, Sur une inégalité entre les intégrales due à Tchebyscheff. Ann. Univ. Mariae Curie-Sklodowska (Poland), A5 (1951), 23-29.

[2] K. Boukerrioua, and A. Guezane-Lakoud, On generalization of Čebyšev type inequalities. J. Inequal. Pure Appl. Math. 8 (2007), no. 2, Article 55, 4 pp.

[3] P. L. Čebyšev, O približennyh vyraženijah odnih integralov čerez drugie. Soobšćenija i protokoly zasedaniı̌ Matemmatičeskogo občestva pri Imperatorskom Har'kovskom Universitete No. 2, 93-98; Polnoe sobranie sočineniı̌ P. L. Čebyševa. MoskvaLeningrad, 1948a, (1882), 128-131.

[4] P. L. Čebyšev, Ob odnom rjade, dostavljajušćem predel'nye veličiny integralov pri razloženii podintegral'nǒ funkcii na množeteli. Priloženi k 57 tomu Zapisok Imp. Akad. Nauk, No. 4; Polnoe sobranie sočineniı̌ P. L. Čebyševa. Moskva-Leningrad, 1948b, (1883),157-169.

[5] M. D. Choi, Positive linear maps on $C^{*}$-algebras. Canad. J. Math. 24 (1972), 520529 .

[6] S. S. Dragomir, On some improvements of Čebyšev's inequality for sequences and integrals, Studia Univ. Babeş-Bolyai, Mathematica (Romania), XXXV (4)(1990), $35-40$. 
[7] S. S. Dragomir, Some improvement of Čebyšev's inequality for isotonic functionals, Atti. Sem. Mat. Fis. Univ. Modena (Italy), 41 (1993), 473-481.

[8] S. S. Dragomir, On the Čebyšev's inequality for weighted means. Acta Math. Hungar. 104 (2004), no. 4, 345-355.

[9] S. S. Dragomir and B. Mond, Some mappings associated with Čebyšev's inequality for sequences of real numbers. Bull. Allahabad Math. Soc. 8/9 (1993/94), 37-55.

[10] S. S. Dragomir and J. E. Pečarić, Refinements of some inequalities for isotonic linear functionals, L'Anal. Num. Théor de L'Approx. (Romania) 18 (1989) (1), 61-65.

[11] S. S. Dragomir and J. Sándor, The Chebyshev inequality in pre-Hilbertian spaces. I. Proceedings of the Second Symposium of Mathematics and its Applications (Timişoara, 1987), 61-64, Res. Centre, Acad. SR Romania, Timişoara, 1988. MR1006000 (90k:46048).

[12] S. S. Dragomir, J. Pečarić and J. Sándor, The Chebyshev inequality in pre-Hilbertian spaces. II. Proceedings of the Third Symposium of Mathematics and its Applications (Timişoara, 1989), 75-78, Rom. Acad., Timişoara, 1990. MR1266442 (94m:46033)

[13] S. S. Dragomir, Inequalities for the Cebyšev functional of two functions of selfadjoint operators in Hilbert spaces. Aust. J. Math. Anal. Appl. 6 (2009), no. 1, Art. 7, 58 pp.

[14] S. S. Dragomir, Cebyšev's type inequalities for functions of selfadjoint operators in Hilbert spaces. Linear Multilinear Algebra 58 (2010), No. 7-8, 805-814.

[15] S. S. Dragomir, Operator Inequalities of the Jensen, Čebyšev and Grüss Type. Springer Briefs in Mathematics. Springer, New York, 2012. xii+121 pp. ISBN: 9781-4614-1520-6.

[16] S. S. Dragomir, A weakened version of Davis-Choi-Jensen's inequality for normalised positive linear maps, Preprint RGMIA Res. Rep. Coll. 19 (2016). Art. 61. [Online http://rgmia.org/papers/v19/v19a61.pdf] .

[17] G. H. Hardy, J. E. Littlewood and G. Pólya, Inequalities, 1st Ed. and 2nd Ed. Cambridge University Press, (1934, 1952) Cambridge, England.

[18] A. Matković, J. Pečarić and I. Perić, A variant of Jensen's inequality of Mercer's type for operators with applications. Linear Algebra Appl. 418 (2006), No. 2-3, 551-564.

[19] D. S. Mitrinović, J. Pečarić and A.M. Fink, Classical and New Inequalities in Analysis, Kluwer Academic, Dordrecht, 1993.

[20] D. S. Mitrinović and J.E. Pečarić, On an identity of D.Z. Djoković, Prilozi Mak. Akad.Nauk. Umj. (Skopje), 12(1)(1991), 21-22.

[21] D. S. Mitrinović and J.E. Pečarić, History, variations and generalizations of the Čebyšev inequality and question of some priorities. II. Rad Jugoslav. Akad. Znan. Umjet. No. 450 (1990), 139-156.

[22] D. S. Mitrinović and P. M. Vasić, History, variations and generalisations of the Čeby̌sev inequality and the question of some priorities. Univ. Beograd. Publ. Elektrotehn. Fak. Ser. Mat. Fiz. No. 461-497 (1974), 1-30.

[23] B. Mond and J. Pečarić, Convex inequalities in Hilbert spaces, Houston J. Math., 19 (1993), 405-420.

[24] B. Mond and J. Pečarić, Classical inequalities for matrix functions, Utilitas Math., 46 (1994), 155-166.

[25] H. R. Moradi, M. E. Omidvar and S. S. Dragomir, An operator extension of $\check{C} e-$ byšev inequality, Preprint RGMIA Res. Rep. Coll. 19 (2016). Art. 81. [Online http://rgmia.org/papers/v19/v19a81.pdf] .

[26] B. G. Pachpatte, New Čebyšev type inequalities involving functions of two and three variables. Soochow J. Math. 33 (2007), no. 4, 569-577.

[27] B. G. Pachpatte, A note on Čebyšev type inequalities. An. Ştiinţ. Univ. Al. I. Cuza Iaşi. Mat. (N. S.)(Romania), 53 (2007), no. 1, 97-102. 
[28] J. Pečarić, Remarks on Biernacki's generalization of Čebyšev's inequality. Ann. Univ. Mariae Curie-Sklodowska Sect. A 47 (1993), 116-122.

[29] J. Pečarić and S. S. Dragomir, Some remarks on Čebyšev's inequality, L'Anal. Num. Théor de L'Approx. 19 (1)(1990), 58-65.

[30] J. Pečarić, T. Furuta, J. Mićić Hot and Y. Seo, Mond-Pečarić Method in Operator Inequalities. Inequalities for Bounded Selfadjoint Operators on a Hilbert Space, Element, Zagreb, 2005.

[31] J. Pečarić, J. Mićić and Y. Seo, Inequalities between operator means based on the Mond-Pečarić method. Houston J. Math. 30 (2004), no. 1, 191-207.

\section{S.S. Dragomir}

Mathematics, College of Engineering \& Science

Victoria University, PO Box 14428

Melbourne City, MC 8001

Australia

School of Computer Science \& Applied Mathematics University of the WitwatersRand Private Bag 3, Johannesburg 2050

South Africa

E-mail address: sever.dragomir@vu.edu.au 